This work is a copy of the published version:

M. Schmidt, T. Pfeiffer, C. Grill, R. Huber, and C. Jirauschek, "Modeling of the Ultra-Stable Operating Regime in Fourier Domain Mode Locked (FDML) Lasers," Proceedings of the 2019 Conference on Lasers and ElectroOptics Europe \& European Quantum Electronics Conference (CLEO ${ }^{\circledR} /$ Europe-EQEC), 23-27 June, 2019, Munich, Germany, paper cj_p_46, doi: 10.1109/CLEOE-EQEC.2019.8873213.

(C2019 IEEE. Personal use of this material is permitted. Permission from IEEE must be obtained for all other uses, in any current or future media, including reprinting/republishing this material for advertising or promotional purposes, creating new collective works, for resale or redistribution to servers or lists, or reuse of any copyrighted component of this work in other works. 


\title{
Modeling of the Ultra-Stable Operating Regime in Fourier Domain Mode Locked (FDML) Lasers
}

\author{
Mark Schmidt $^{1}$, Tom Pfeiffer ${ }^{2}$, Christin Grill $^{2}$, Robert Huber $^{2}$, Christian Jirauschek $^{1}$ \\ 1. Department of Electrical and Computer Engineering, Technical University of Munich, Arcisstraße 21, 80333 Munich, Germany \\ 2. Institut für Biomedizinische Optik, Universität zu Lübeck, Peter-Monnik-Weg 4, 23562 Lübeck, Germany
}

Fourier domain mode locked (FDML) fiber lasers are broadband wavelength-swept ring systems with record sweep speeds. Lasing is achieved by synchronizing the roundtrip time of the optical field in the fiber delay cavity with the sweep period of a tunable Fabry-Pérot (FP) bandpass filter. Since their invention in 2006, FDML lasers have dramatically enhanced the capabilities of optical coherence tomography (OCT) and various sensing applications. However, the physical coherence limits, such as the maximum achievable coherence length, are yet unknown. An important breakthrough in reaching this limit is a recently experimentally demonstrated highly coherent operation mode over a bandwidth of more than $100 \mathrm{~nm}$ [1], referred to as the sweet spot. The sweet spot operation mode is characterized by nearly shot-noise limited fluctuations in the intensity trace of the laser with significantly enhanced coherence properties, whereas in conventional FDML laser systems the intensity trace is distorted by high frequency noise which negatively affects the coherence length. This ultra-low noise operating regime was generated by an almost perfect compensation of the fiber dispersion with a manually fine tuned chirped fiber Bragg grating and a highly synchronized sweep rate of the FP filter with an accuracy in the range of $\mathrm{mHz}$. Polarization effects were controlled with a polarization maintaining semiconductor optical amplifier (SOA) gain medium and a polarization controller.
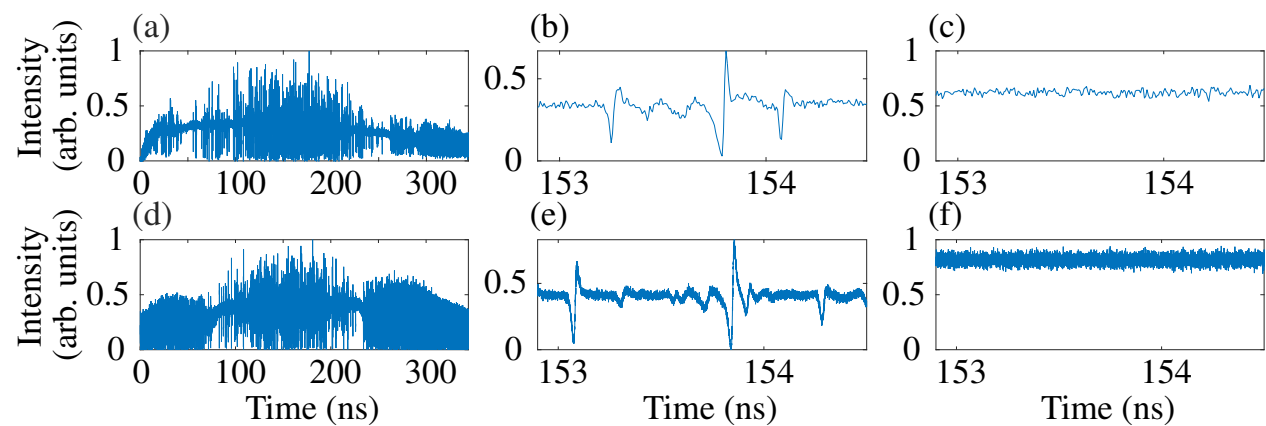

$$
\text { (e) }
$$

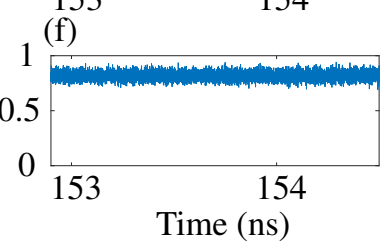

Fig. 1 (a) Measured intensity trace of a non-dispersion compensated FDML laser (104 nm backward sweep, center wavelength $1307 \mathrm{~nm}$ ), recorded with an analog bandwidth of $50 \mathrm{GHz}$. (b) Zoom into the intensity trace of (a) to demonstrate the hole dynamics. (c) Sweet spot operation mode for comparison. (d)-(f) Simulation results for the experimental scenarios in (a)-(c).

In order to gain a theoretical understanding of the sweet spot operation mode we developed a theoretical framework based on our previous FDML laser model [2,3] with an improved description of the SOA gain medium. This model is able to fully describe the FDML laser dynamics in a polarization maintaining setup as can be observed from Fig. 1 where a measured intensity trace in (a) and (b) of a non-dispersion compensated laser is compared to a numerical solution of the same setup in (d) and (e). In particular, the noise evolution mainly caused by the timing mismatches induced by the fiber dispersion can be accurately reproduced. A dominant source of noise are so-called holes [4] which correspond to dips in the intensity trace of up to $100 \%$ followed by an overshoot due to the SOA gain recovery as can be seen in Fig. 1 (b) and (e). Their occurrence is irregular and the noise pattern is not stable over consecutive roundtrips. Further simulations show that the sweet spot operation can form even in non-ideally synchronized systems as presented in Fig. 1 (c) and (f) which are of practical relevance. Our simulation model reveals that the frequency dependent group delay of the moving FP filter can stabilize accumulative perturbations in the ring system, e.g. a residual fiber dispersion with a maximum group delay difference of a few $100 \mathrm{fs}$ or a non-synchronized sweep frequency in the range of $\mathrm{mHz}$, such that no holes are formed in the intensity trace. We are convinced that our findings are essential for a further development of high coherence FDML lasers. Furthermore, the feasibility of FDML frequency combs and the generation of Fourierlimited pulses by sweep compression can be addressed within our model.

\section{References}

[1] T. Pfeiffer, M. Petermann, W. Draxinger, C. Jirauschek, and R. Huber, "Ultra low noise Fourier domain mode locked laser for high quality megahertz optical coherence tomography," Biomed. Opt. Express 9, 4130-4148 (2018).

[2] C. Jirauschek, B. Biedermann, and R. Huber, “A theoretical description of Fourier domain mode locked lasers,” Opt. Express 17, 2401324019 (2009).

[3] C. Jirauschek and R. Huber, "Modeling and analysis of polarization effects in Fourier domain mode-locked lasers," Opt. Lett. 40, 2385$2388(2015)$

[4] S. Slepneva, B. O’Shaughnessy, A. Vladimirov, S. Rica, and G. Huyet, “Turbulent laser puffs,” arXiv preprint arXiv:1707.08304 (2017). 